\title{
Management of retinal detachment with severe proliferative vitreoretinopathy in Terson syndrome - case report
}

\author{
Katarzyna Nowomiejska, Edyta Koman, Robert Rejdak \\ Department of General Ophthalmology, Medical University of Lublin, Poland
}

\begin{abstract}
We present a case of aneurysmal subarachnoid hemorrhage accompanied by a dense vitreous hemorrhage and subsequent retinal detachment with strong proliferative retinopathy (PVR) in a young woman. The surgical treatment included vitrectomy with PVR removal, ILM peeling and silicone oil as a tamponade. In our case, the treatment resulted in recovery of vision and improved activities of daily living, although unusually vitreous hemorrhage was complicated with retinal detachment and strong PVR.
\end{abstract}

KEY WORDS: Terson syndrome, retinal detachment, vitrectomy

Ophthalmol J 2017; Vol. 2, No. 4, 113-116

\section{INTRODUCTION}

Terson syndrome is defined as association of vitreous, subhyaloid (preretinal) or retinal haemorrhage with aneurysmal subarachnoid haemorrhage (SAH) or head trauma [1] in patients without other ocular pathology. The pathogenesis of Terson syndrome remains unclear [2]. Patients with subarachnoid hemorrhage or subdural hemorrhage often present cognitive impairment which hinders accurate diagnosis. Blood may enter into the vitreous cavity around the retinal vessels near the optic disc, inside the eye. The blood may spread intraretinally, sub-ILM, or along the retinal vessels [3]. Terson syndrome is named by a French ophthalmologist Albert Terson who published an article in 1900 [4]. Intraocular haemorrhages of different types have been documented in 8-20\% of individuals with $\mathrm{SAH}[5,6]$. Terson syndrome represents $5.5 \%$ of all non-diabetic and non-traumatic vitreous haemorrhages [7].

In cases of minor bleeding spontaneous resorption may occur and vision is restored. In long-stand- ing vitreous hemorrhage complications such as epiretinal membrane [8] and retinal detachment may occur and can lead to loss of vision or serious visual impairment [9].

The surgical procedure of choice in Terson syndrome is 20 or 23 gauge pars plana vitrectomy (PPV) [1]. We describe a case of retinal detachment with severe proliferative vitreoretinopathy (PVR) trated with vitrectomy and internal limiting membrane (ILM) peeling.

\section{CASE DESCRIPTION}

Thirty-nine year old female presented with the haemorrhage into the vitreous cavity of the right eye as a result of cerebral aneurysm rupture of the right pericallosall artery. She inderwent a cerebral aneurysm clipping, Visual acuity was light perception in her right eye and 1.0 in the left eye. Fundus examination and ultrasonogrphy revealed vitreous hemorrhage in the right eye. One month later 23 gauge vitrectomy was performed in the right eye 


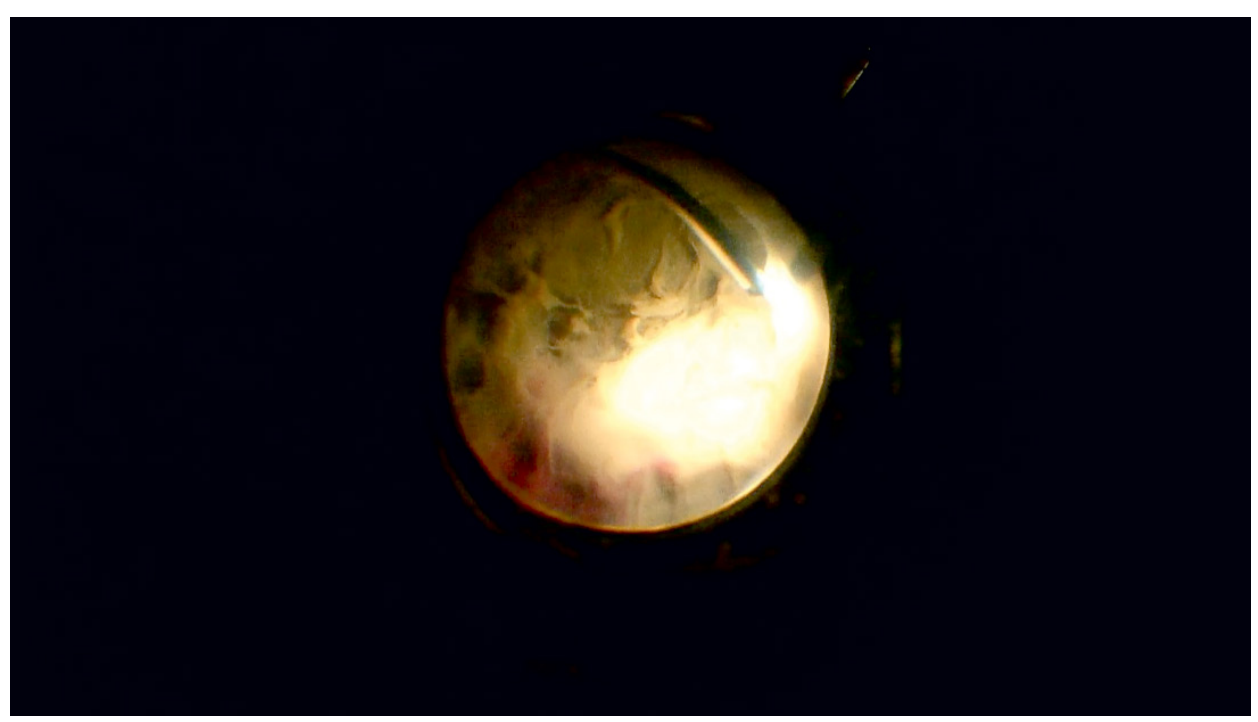

FIGURE 1. Dense vitreous hemorrhage at the beginning of the vitrectomy

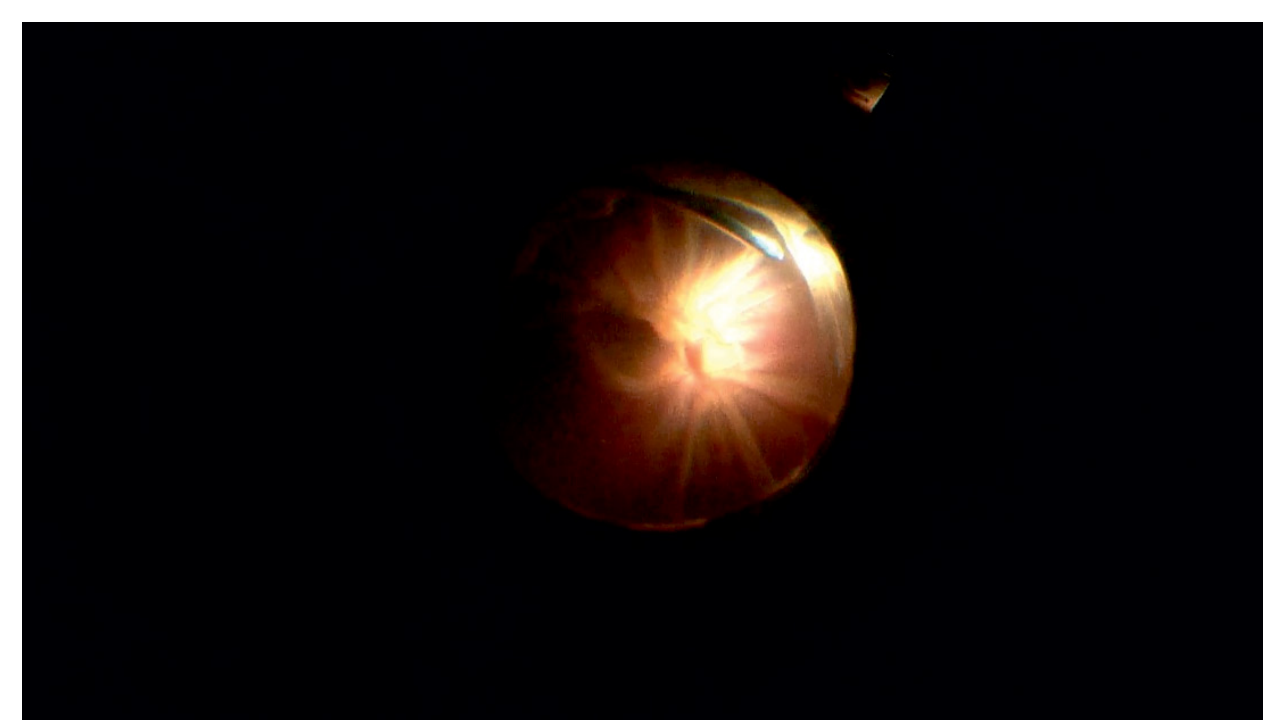

FIGURE 2. Retina at the posterior pole schrinked with advanced proliferative vitreoretinoapthy

with lens sparing. After removing dense vitreous hemorrhage (Fig. 1) schrinkage in the posterior pole was observed (PVR D1) (Fig. 2). Epiretinal PVR was removed with Eckart forceps and the retina was flattened with perfluorodecalin (Fig. 3). Internal limiting membrane peeling under perfluorodecain was done after staining with indocyanine green (Fig. 4). After fluid-air exchange and removing of perfluorodecalin, $5000 \mathrm{cSt}$ silicone oil was given as a tamponade. The visual acuity was 0.1 with $+3 \mathrm{D}$ lens. Recovery of vision improved activities of daily living of the patient. Six months later phacoemulsification with intraocular lens implantation (Fig. 5) and silicon oil removal (Fig. 6). The visual acuity is still 0.1 , but without correction.

\section{DISCUSSION}

In this paper we present a rare case of vitreous hemorrhage in Terson syndrome complicated by retinal detachment.

In general, the results of surgical treatment of Terson syndrome with PPV are very good and this surgical procedure provides generally good visual recovery (more than 0.5$)[10,3,5]$. Some authors state that PPV leads to rapid recovery irrespectively of the timing of vitrectomy or of preoperative visual acuity (nararayan). There is a success rate of $90 \%$ in a single surgery (garweg). In our case two vitrectomies were performed to achieve the final outcome.

The most common complication of the vitreous hemorrhage in Terson syndrome is formation of 


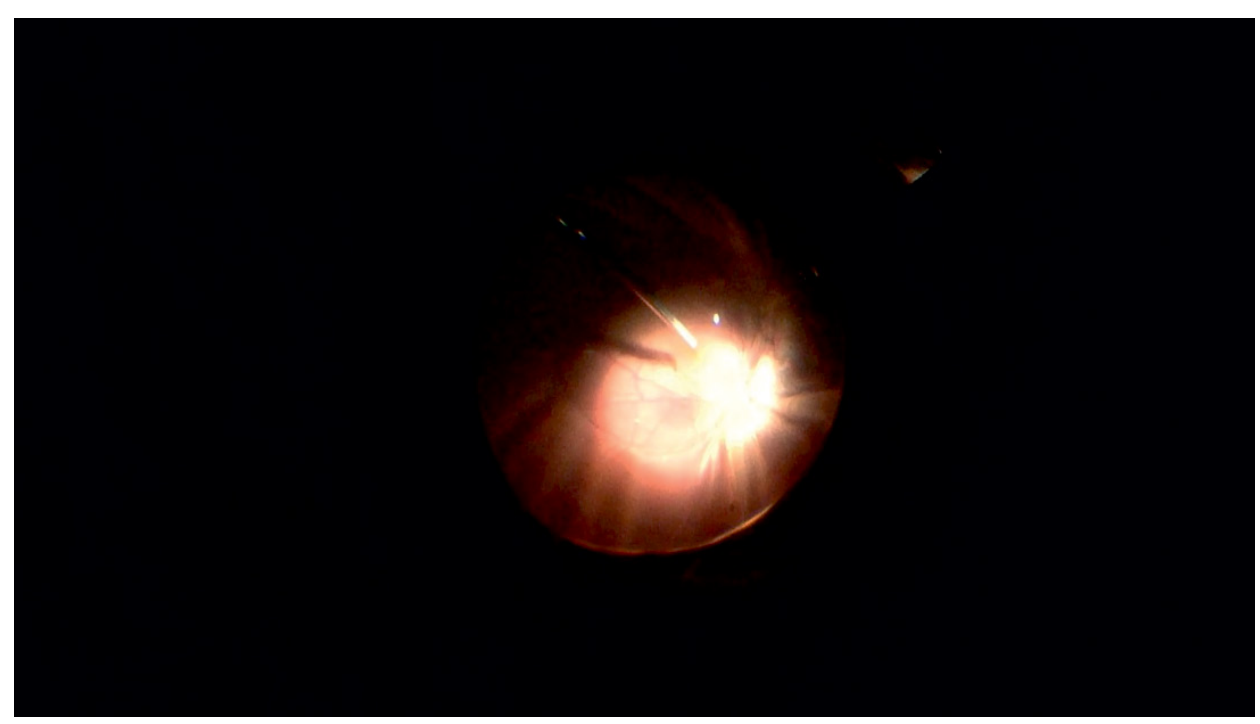

FIGURE 3. Flattening of the retina with perfluorodecalin after removing epiretinal proliferative vitreoretinopathy



FIGURE 4. Internal limiting membranę peeling under perfluorodecalin after staining with indocyanine green



FIGURE 5. Lens removal during the second vitrectomy epiretinal membranes, observed in approximately 16-59\% of cases [11]. Strong PVR as a complication is quite rare in Terson syndrome. In the study of Narayanan there were 3 retinal detachments in a group of 28 eyes with Terson syndrome following brain injury [1]. Retinal detachment as a complication of Terson sydrome was described for the first time in 1994 by Velikay and co-authors [12]. The authors report five eyes from four patients with Terson syndrome due to spontaneous aneurysm rupture, in whom retinal detachment with PVR developed. The retina in all eyes could be reattached. 


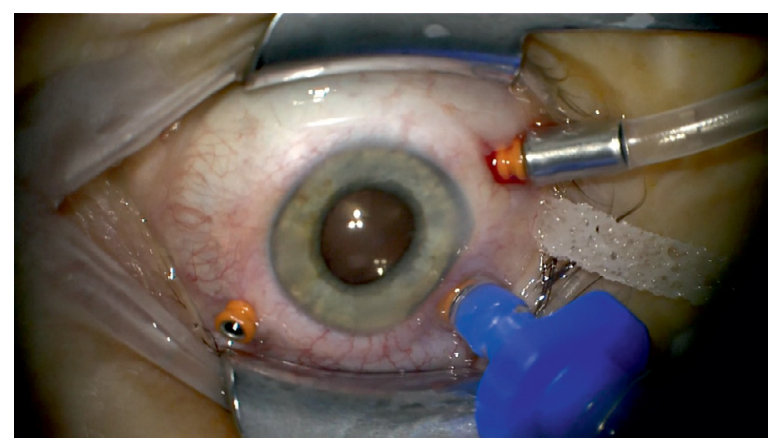

FIGURE 6. Silicone oil removal during the second vitrectomy

Other complications of a long-lasting vitreous hemorrhage include: decline of retinal pigment epithelium, atrophy the optic nerve, macular hole, retinal folds, cysts of the retina, cataract [13]. The incidence of intraocular pathologies has been found to be significantly higher in patients who lost consciousness at the onset of SAH [14]. Another finding that can be seen intraoperatively is perimacular ring [11]. It is a consequence of a preretinal or retrohyaloideal haemorrhage.

PVR is a cellular reaction representing a vitreo-retinal wound healing response characterized by formation of periretinal non-vascular fibrocellular membranes [15]. PVR develops as a relatively rare complication in about $8-10 \%$ of patients with primary retinal detachment. Evolving of PVR in long-existing haemorrhage may be caused by toxic effects of iron from red blood cells [16]. Intraocular bleeding can lead to proliferation of fibroblasts or glial cells. Posttraumatic vitreous hemorrhage is also strongly linked to less favorable outcomes and PVR development [17]. Close follow-up with regular B-scan ultrasonography examinations is recommended to detect PVR early preoperatively [13].

The surgical management of strong PVR is the same as in long-lasting rhematogenous retinal detachment. If needed retinotomy is performed but it was not necessary in our case. The Silicone Study concluded that $\mathrm{C} 3 \mathrm{~F} 8$ gas or silicone oil is more effective than SF6 gas in the treatment of PVR cases [18].

In summary, our study provides additional knowledge to the clinical picture of the Terson syndrome. Retinal detachment with PVR can be observed in the course of Terson syndrome and it requires removal of fibrocellular membranes, ILM peeling and silicone oil as a tamponade.

\section{REFERENCES}

1. Narayanan R, Taylor SC, Nayaka A, et al. Visual Outcomes after Vitrectomy for Terson Syndrome Secondary to Traumatic Brain Injury. Ophthalmology. 2017; 124(1): 118-122, doi: 10.1016/j.ophtha.2016.09.009, indexed in Pubmed: 27817917.

2. Ogawa T, Kitaoka T, Dake Y, et al. Terson syndrome: a case report suggesting the mechanism of vitreous hemorrhage. Ophthalmology. 2001; 108(9): 1654-1656, doi: 10.1016/s0161-6420(01)00673-x, indexed in Pubmed: 11535467.

3. Michalewska Z, Michalewski J, Nawrocki J. Possible methods of blood entrance in Terson syndrome. Ophthalmic Surg Lasers Imaging. 2010; 41 Suppl: S42-S49, doi: 10.3928/15428877-20101031-15, indexed in Pubmed: 21117599.

4. Terson A. De l'hémorrhagie dans le corps vitre au cours de l'hémorrhagie cerebrale. Clin Ophthalmol. 1900; 6: 309-123.

5. Kuhn F, Morris R, Witherspoon CD, et al. Terson syndrome. Results of vitrectomy and the significance of vitreous hemorrhage in patients with subarachnoid hemorrhage. Ophthalmology. 1998; 105(3): 472-477, doi: 10.1016/S0161-6420(98)93030-5, indexed in Pubmed: 9499778.

6. Nowosielska A, Czarnecki W, Zabek M. [Terson syndrome in a course of cerebral aneurysm--clinical assessment]. Klin Oczna. 2003; 105(12): 49-51, indexed in Pubmed: 12866172.

7. Verbraeken H, Van Egmond J. Non-diabetic and non-oculotraumatic vitreous haemorrhage treated by pars plana vitrectomy. Bull Soc Belge 0phtalmol. 1999; 272: 83-89, indexed in Pubmed: 10427924.

8. Errera MH, Barale PO, Ounnoughene $\mathrm{Y}$, et al. [25-Gauge transconjunctival vitrectomy in a case of bilateral epiretinal membrane associated with a Terson syndrome]. J Fr Ophtalmol. 2009; 32(4): 268-272, doi: 10.1016/j.jfo.2008.12.007, indexed in Pubmed: 19769858.

9. Shaw HE, Landers MB, Sydnor CF. The significance of intraocular hemorrhages due to subarachnoid hemorrhage. Ann Ophthalmol. 1977; 9(11): 1403-1405, indexed in Pubmed: 931282.

10. Sharma T, Gopal L, Biswas J, et al. Results of vitrectomy in Terson syndrome. Ophthalmic Surg Lasers. 2002; 33(3): 195-199, indexed in Pubmed: 12027098.

11. Garweg JG, Koerner F. Outcome indicators for vitrectomy in Terson syndrome. Acta Ophthalmol. 2009; 87(2): 222-226, doi: 10.1111/j.17553768.2008.01200.x, indexed in Pubmed: 18537934.

12. Velikay M, Datlinger $P$, Stolba U, et al. Retinal detachment with severe proliferative vitreoretinopathy in Terson syndrome. Ophthalmology. 1994; 101(1): 35-37, doi: 10.1016/s0161-6420(13)31240-8, indexed in Pubmed: 8302561.

13. Ritland JS, Syrdalen P, Eide N, et al. Outcome of vitrectomy in patients with Terson syndrome. Acta Ophthalmol Scand. 2002; 80(2): 172-175, doi: 10.1016/s0161-6420(13)31240-8, indexed in Pubmed: 11952484.

14. Obuchowska I, Turek G, Mariak Z, et al. Early Intraocular Complications of Subarachnoid Haemorrhage after Aneurysm Rupture. Neuroophthalmology. 2014; 38(4): 199-204, doi: 10.3109/01658107.2014.911918, indexed in Pubmed: 27928299.

15. Wiedemann $P$, Yandiev Y, Hui YN, et al. Pathogenesis of Proliferative Vitreoretinopathy. Retina. 2013: 1640-1646, doi: 10.1016/b978-14557-0737-9.00097-7.

16. Swallow CE, Tsuruda JS, Digre KB, et al. Terson syndrome: CT evaluation in 12 patients. AJNR Am J Neuroradiol. 1998; 19(4): 743-747, indexed in Pubmed: 9576666.

17. Acar U, Tok OY, Acar DE, et al. A new ocular trauma score in pediatric penetrating eye injuries. Eye (Lond). 2011; 25(3): 370-374, doi: 10.1038/eye.2010.211, indexed in Pubmed: 21252953.

18. Abrams GW, Azen SP, McCuen BW, et al. The incidence of corneal abnormalities in the Silicone Study. Silicone Study Report 7. Arch Ophthalmol. 1995; 113(6): 764-769, doi: 10.1001/ archopht.1997.01100150337005, indexed in Pubmed: 7786219. 